\section{Violence and schizophrenia: examining the evidence}

\author{
ELIZABETH WALSH, ALEC BUCHANAN and THOMAS FAHY
}

\begin{abstract}
Background It is now accepted that people with schizophrenia are significantly more likely to be violent than other members of the general population. A less acknowledged fact is that the proportion of societal violence attributable to schizophrenia is small.
\end{abstract}

\begin{abstract}
Aims To critically examine the epidemiological evidence for the association between violence and schizophrenia and estimate the impact of this association on society.
\end{abstract}

\begin{abstract}
Method A selective review of the key literature on the epidemiology of violence and schizophrenia. Population-attributable risks for violence in schizophrenia are calculated from population-based studies.
\end{abstract}

Results Most studies confirm the association between violence and schizophrenia. Recent good evidence supports a small but independent association. Comorbid substance abuse considerably increases this risk. The proportion of violent crime in society attributable to schizophrenia consistently falls below $10 \%$.

\section{Conclusions Less focus on the relative risk and more on the absolute risk of violence posed to society by people with schizophrenia would serve to reduce the associated stigma. Strategies aimed at reducing this small risk require further attention, in particular treatment for substance misuse.}

Declaration of interest E.W. was funded by a WellcomeTraining Fellowship.
The conclusions of those reaching the putative link between schizophrenia and violence changed in the late twentieth century. Until the early 1980s the consensus was that those with schizophrenia were no more likely than the general population to be violent. $\mathrm{N}$ ew epidemiological evidence has emerged, however, that has radically challenged this view. It is now generally accepted that people with schizophrenia, albeit by virtue of the activity of a small subgroup, are significantly more likely to be violent than members of the general population, but the proportion of societal violence attributable to this group is small. This review provides an overview of the main studies that have influenced current thinking about the association, followed by an epidemiological appraisal of the difficulties inherent in this type of research. It attempts to differentiate those most at risk of behaving violently and concludes with some estimate of the absolute risk posed to the community by those with schizophrenia.

\section{METHOD}

Computerised M edline and Psycinfo searches were performed from January 1990 to December 2000 using the terms VIOLENCE, ASSAULT, SCHIZOPHRENIA, SEVERE MENTAL ILLNESS, MAJOR MENTAL DISORDER and PSYCHOSIS. We wished to conduct a critique of the main epidemiological studies that have established the link between the risk of violence and schizophrenia. We focus on these and on some older studies to demonstrate the differing methodologies employed in this type of research. This is not an all-inclusive review, and the choice of articles reflects the authors' qualitative assessment of current themes of importance in this area of research. For additional reviews, see Mullen (1997) and Eronen et al, (1998).

\section{VIOLENCE STUDIES}

Three different approaches have been used to examine the association between schizophrenia and violence. These include studies estimating the prevalence of:

(a) violent acts in those with schizophrenia;

(b) schizophrenia in individuals who have committed violent acts;

(c) violence in those with and without schizophrenia, regardless of involvement with the mental health or criminal justice systems (community-based epidemiological studies).

\section{Studies estimating the prevalence of violent acts among those with schizophrenia}

Two main designs have been used: crosssectional studies and cohort studies using case linkage technology.

\section{Cross-sectional studies}

With violence being a main selection criterion for admission, studies of violence committed before and during hospitalisation are of limited usefulness because they will overestimate any association. Discharged patients are a selected group because they are generally judged not to pose a threat or to pose less threat than those retained in hospital. As such, one would expect lower rates of violence to be recorded at this time than prior to admission.

Before hospitalisation. Humphreys et al (1992) estimated that $20 \%$ of first-admission patients with schizophrenia had behaved in a life-threatening manner prior to admission. Volavka et al (1997) estimated that $20 \%$ of first-contact patients with schizophrenia had assaulted another person at some time in the past.

During hospitalisation. These studies have suggested relatively high rates of assaultativeness (Karson \& Bigelow, 1987; Walker \& Seifert, 1994). Results must be viewed with particular caution, however, because violence may be more of a response to the contextual setting of a confined ward than to an individual's mental state.

Following discharge. The two most comprehensive studies published to date on violence risk after discharge fail to provide separate data for schizophrenia (Steadman et al, 1998; Link et al, 1992, see 
below). M onahan \& Applebaum (2000), as part of the M acArthur Risk Assessment Study, estimated the prevalence of community violence in discharged patients by diagnosis. Violence was measured from multiple sources every 10 weeks for a year. Of the $17 \%$ of patients with a diagnosis of schizophrenia, $9 \%$ were violent in the first 20 weeks after discharge. This compares with a violence prevalence of $19 \%$ for depression, $15 \%$ for bipolar disorder, $17.2 \%$ for other psychotic disorders, $29 \%$ for substance misuse disorders and $25 \%$ for personality disorder alone. The fact that this and other studies have found rates of violence to be lower in those with schizophrenia than in those with other diagnoses ( $\mathrm{H}$ arris et al, 1993; Wallace et al, 1998) should not be misinterpreted to suggest that schizophrenia may be irrelevant or even a protective factor against violence. It is probably true that schizophrenia is less of a violence risk than substance misuse, personality disorder and possibly other mental disorders, but when compared with the general population, as this review amply demonstrates, the evidence is overwhelmingly in favour of an increased risk of violent behaviour.

\section{Retrospective cohorts using case linkage}

Three studies using slightly different methodologies have drawn similar conclusions. In the first, 644 patients with schizophrenia followed for up to 15 years on a police register, were found to be four times more likely to have committed a violent crime than the general population (Lindqvist \& Allebeck, 1990).

The second study compared the rate of criminal convictions among 538 incident cases of schizophrenia with that of nonpsychotic psychiatric controls matched for age and gender (W essely et al, 1994). M ale patients with schizophrenia were twice as likely as men with other mental disorders to have a violent conviction. This was despite the control group containing a substantial minority of individuals with psychiatric disorders with an established association with crime. Women with schizophrenia were also significantly more likely to be convicted of violent crime than controls.

In the third study, M ullen et al (2000), in Australia, studied two groups of patients with schizophrenia first admitted in either 1975 (before major deinstitutionalisation) or 1985 (when community care was becoming the norm). Compared with general population controls, both groups were significantly more likely to be convicted for all categories of criminal offending, except sexual offences. Those with comorbid substance abuse accounted for a disproportionate level of offending. The increased number of convictions in those with schizophrenia in the 1985 group compared with the 1975 group seemed to reflect a general increase in offending in those of a similar age, gender and place of residence. As such, the shift to community care was not marked by any significant change in relative rates of conviction in schizophrenia. The effect of community care on risk of violence in schizophrenia requires further study. O ne study examining homicide statistics in the UK has reported little fluctuation in the numbers of people with mental illness committing homicide between 1957 and 1995 and a $3 \%$ annual decline in their contribution to the official statistics (Taylor \& Gunn, 1999).

\section{Unselected birth cohort studies}

Hodgins (1992), in a 30-year follow-up of an unselected Swedish birth cohort, found that compared with those with no mental disorder, males with major mental disorder had a 4-fold and women a 27.5-fold increased risk of violent offences. N o separate data were provided for schizophrenia. A later study using the same methodology revealed similar findings ( $\mathrm{H}$ odgins et al, 1996).

The first cohort study to demonstrate the quantitative risk of violent behaviour for specific psychotic categories followed an unselected birth cohort of 12058 individuals prospectively for 26 years (Tiihonen et al, 1997). The risk of violent offences among males with schizophrenia was 7-fold higher than controls without mental disorder.

Brennan et al (2000) traced all arrests for violence and hospitalisations for mental illness in a birth cohort followed to age 44 years. Schizophrenia was the only major mental disorder associated with increased risk of violent crime in both males and females, adjusting for socio-economic status, marital status and substance abuse.

Arseneault et al (2000) studied the pastyear prevalence of violence in 961 young adults who constituted $94 \%$ of a total city birth cohort. Three Axis I disorders were uniquely associated with violence after controlling for demographic risk factors and all other comorbid disorders: alcohol dependence, marijuana dependence and schizophrenic spectrum disorder.

\section{Studies estimating the prevalence of schizophrenia in individuals who have committed violent acts}

$\mathrm{N}$ umerous studies have estimated the prevalence of schizophrenia among prison inmates. Despite problems of unstandardised diagnoses and the frequent absence of comparison data among the general population, the evidence suggests an over-representation of those with schizophrenia among offender populations.

Taylor \& Gunn (1984), using validated diagnoses, studied the psychiatric status of male prisoners remanded to a prison in south London. $\mathrm{N}$ ine per cent of those subsequently convicted of non-fatal violence and $11 \%$ convicted of fatal violence had schizophrenia, which are substantially higher prevalences than would have been expected in the general population for the same area (0.1-0.4\%).

Teplin (1990) compared the prevalence of schizophrenia among 728 male prisoners with that of the general population. The prevalence in the jail population $(2.7 \%)$ was found to be three times higher than that of the general population $(0.91 \%)$ after controlling for socio-demographic factors.

Eronen et al (1996), in a study of 693 people convicted of homicide in Finland, found schizophrenia to be associated with an 8-fold increase in homicide by men and a 6.5 -fold increase by women.

W allace et al (1998), in a study of individuals convicted of serious offences in Victoria County, Australia, searched for evidence of a psychiatric contact on the county psychiatric register. Those with schizophrenia were found to be over four times more likely to be convicted of interpersonal violence and ten times more likely to be convicted of homicide than the general population.

\section{Community prevalence studies}

The above studies, although valuable in making inferences about the relationship between violence and schizophrenia, are subject to biases that will be discussed 
below. Data on unselected samples of people from the open community are needed to augment the findings. Probably the most important study in the violence literature to date is that of Swanson et al (1990). Using a sample of 10059 adult residents from Epidemiologic Catchment A rea (ECA) study sites (Eaton \& Kessler, 1985), the authors examined the relationship between violence and psychiatric disorder. Eight per cent of those with schizophrenia alone were violent, compared with $2 \%$ of those without mental illness. Comorbidity with substance abuse increased this percentage to $30 \%$.

Two other community epidemiological studies, both finding increased risk of violence among psychiatric patients (Link et al, 1992) and those with major mental disorder (Stueve \& Link, 1997), respectively, failed to provide data on schizophrenia as a separate diagnostic entity.

\section{METHODOLOGICAL LIMITATIONS OF VIOLENCE STUDIES}

The majority of studies over the past two decades have demonstrated a statistical association between schizophrenia and violence. It can be argued, therefore, that the accumulated evidence from studies adopting different methodologies supports a causal relationship, because the consistency of findings across studies overshadows the methodological weaknesses of any one. Some have argued against this conclusion (Arboleda-Florez et al, 1998), suggesting that this overlooks the possibility of consistent design flaws, including violence measurement, selection bias, confounding and poorly controlled comparisons, which may offer rival explanations for the current statistical associations. It is thus important that the findings of each study be appraised critically in the light of the limitations inherent in research of this complexity.

A brief overview of each of these limitations from an epidemiological viewpoint is outlined below.

\section{Definition and measurement of exposure}

Some studies include schizophrenia as part of a heterogeneous group of psychotic disorders (Hodgins, 1992; Hodgins et al, 1996; Steadman et al, 1998) or do not give the diagnostic breakdown of subjects at all (Link et al, 1992). Fewer examine schizophrenia alone (Lindqvist \& Allebeck, 1990; Wessely et al, 1994) and those that do use varying diagnostic techniques. Diagnoses are variously derived from case notes, psychiatric registers, clinical interviews or research interviews. Case-note diagnoses are dependent on individual clinical judgements. Those extracted from case registers are usually those made at discharge and are subject to the same limitations. These diagnoses may be more reliable, however, than those made at a single clinical interview because they are usually based on a period of observation in hospital, collateral information and previous history, which are likely to increase the validity of diagnoses. The use of one agreed diagnostic procedure in studies would allow comparisons of like with like.

\section{Definition and measurement of outcome}

How violence is defined varies greatly and reported rates differ, depending on the levels of violence measured. Unsurprisingly, studies that include threats as well as physical contact record higher rates than those that include contact alone. It is virtually impossible to find violence defined in the same way in any two studies by different researchers. This highlights the need for the development of a standardised, validated, reliable and acceptable rating instrument that could be adopted across studies.

$M$ easurement of violence in studies has relied upon different single (self-report, informant, case notes, official records) or combined sources of information. All sources have inherent limitations. Selfreport measures may underreport violence because of the desire for social acceptability or fear of adverse consequences of reporting. Additionally, retrospective designs produce problems with recall of sometimes distant events. Informants, who are often nominated by patients, may not be the most suitable people to provide information or be aware of incidents. Case notes are of limited usefulness because they are often incomplete. With regard to police contacts or arrest records, the proportion of violent acts that leads to arrest and prosecution varies as a function of the intensity and quality of policing, behaviour of the suspect, the availability of diversion to the mental health systems and the severity of the offence.

Records of criminal convictions are a widely used data source across studies. $M$ ost violent individuals are not convicted (Elliott et al, 1986). The mentally ill tend to be diverted to the mental health care system at various stages from apprehension to conviction. As such, it is likely that only the more serious crimes will lead to conviction. For this reason, the association between schizophrenia and more minor forms of violence is impossible to estimate from this source. For more serious offending such as homicide, individuals are more likely to be brought to trial and convicted, thus justifying the dependence on criminal registers. Unfortunately, as with all such registers, they are prone to data errors, are not inclusive of all convictions and often relate to one geographical area, taking no account of crimes committed outside that jurisdiction.

The more recent use of multiple combined measures for violence has highlighted the limitations of the majority of previous studies that relied on a single source. Steadman et al (1998) used agency records, selfreport and collateral informants to collect information on violent acts. The one-year period prevalence for violence was $4.5 \%$ using agency records (arrest and rehospitalisation records) alone, $23.7 \%$ by adding patient self-reported acts that had not been in agency records and $27.5 \%$ by adding collateral informant-reported acts that had not been in either agency records or patient self-reports. Thus, the final prevalence was six times higher than it would have been if estimated from agency records alone. Mulvey et al (1994a) specifically set out to compare the yield of violence when different sources were used. A dramatically different picture emerged, depending on the source. These results support the previous observation that self-report methods consistently produce a higher frequency of violence than official records (Elliott et al, 1986). Thus, to provide accurate empirical data, it is crucial that it be based on self-report in conjunction with collateral informant and official records. O ne problem inherent to the use of multiple measures is that judgement must be made about what constitutes a single episode of violence and how the inconsistencies that may exist between reports should be handled. 


\section{Bias}

Selection bias can occur whenever the identification of individual subjects for inclusion into a study, on the basis of either exposure or outcome status, depends in some way on the other axis of interest. This bias will result in an observed relationship between exposure (schizophrenia) and outcome (violence) that is different among those who are entered into the study than among those who would have been eligible but did not participate. For example, a psychotic individual's refusal to participate in a study or follow-up interviews might be related to his or her propensity for violence. If so, the rates of violence for those included in the samples may be lower than the true rates for individuals with schizophrenia.

Location of recruitment is a crucial factor in interpreting any such association. Research on violence and mental illness is dominated by data on hospitalised/ discharged patients, but most individuals with mental disorder are not hospitalised (Robins \& Reiger, 1991). Cross-sectional prevalence studies in representative samples of community residents with both treated and untreated mental disorders largely overcome the problem of selection bias, although not completely. They frequently exclude those in jail (Steadman et al, 1998) and, as such, will underestimate any association.

It is not unusual to find high refusal and attrition rates in these studies, also leading to selection bias. In one study, only $50 \%$ of subjects completed all five followup interviews. These compliant subjects were found to be significantly less likely to have a history of previous violence - a major predictor of future violence - than those lost to follow-up (Steadman et al, 1998).

In analytical studies, the risk of violent offending in cases is expressed relative to the risk in controls. It is thus important that the results be interpreted with specific reference to the control group chosen. If, for example, risk of offending in schizophrenia is estimated relative to non-psychotic psychiatric controls (W essely et al, 1994), the risk ratio will depend on whether or not that group contains an excess of patients with personality disorder and substance abuse disorders, both of which are linked to violent behaviour. If national or populationbased figures are used for comparison, they may not take into account the confounding effect of social class on violence (W allace et al, 1998). Alternatively, if neighbourhood controls are chosen, the estimated risk may not be generalisable to the population at large (Steadman et al, 1998).

Other possible biases include interviewer bias and recall bias. On reading most violence studies it is unclear whether interviewers were blind to subject status. If not, selective probing for symptoms of mental illness and/or violent episodes may result in interviewer bias.

\section{Confounding}

A confounder is a factor that is associated with the exposure (schizophrenia) and, independent of this exposure, is a risk factor for outcome (violence). Additionally, it should not be on the causal pathway between exposure and outcome. Statistical relationships observed between schizophrenia and violence in any particular study will hinge on the investigator's understanding and statistical treatment of confounding factors (Arboleda-Florez et al, 1998). Because of the uncertainty of the causal pathway between schizophrenia and violence, it is unclear what variables should be considered as confounders. The more robust studies do control for a range of possible confounding factors, but these are by no means uniform. The relationship is even more complex than this, however, with a wide range of personal and situational factors that must be important in the mediation of violence being impossible to measure.

\section{PREDICTORS OF VIOLENT BEHAVIOUR IN SCHIZOPHRENIA}

Risk factors for violence that operate in those without mental illness operate in schizophrenia, with strong predictors including a history of previous violence and substance abuse. H owever, no sizeable body of evidence clearly indicates the relative strength of schizophrenia or mental illness in general as a risk factor for violence compared with other risk factors (M ulvey et al, 1994b). Indeed, compared with the magnitude of risk associated with the combination of male gender, young age and lower socio-economic status, the risk of violence presented by mental disorder is modest (M onahan, 1997).

Two factors appear to discriminate those with schizophrenia at increased risk of committing violent acts: comorbid substance abuse and acute psychotic symptoms.

It has been demonstrated repeatedly that schizophrenia with comorbid substance abuse increases the risk of violence considerably compared with schizophrenia without comorbidity (Swanson et al, 1990; Cuffel et al, 1994; Tiihonen et al, 1997; W allace et al, 1998). It is important to note that because there is an increase in violence risk in those without comorbidity, substance abuse merely increases the level of risk rather than causing it (Arsenault et al, 2000; Brennan et al, 2000). Hence, the risk from substance abuse appears to be additive.

With regard to acute symptomatology, Taylor estimated that $46 \%$ of a sample of psychotic offenders were definitely or probably driven by delusions (Taylor, 1985). But delusions are an extremely common psychopathological phenomenon in psychosis and serious violence is not, so other factors must be operating (Taylor, 1998).

In a methodologically robust study, Link et al (1992) compared arrest rates and self-reported violence in a sample of community residents with no history of psychiatric contact with current and former patients with heterogeneous diagnoses from the same area. Former patients invariably were more violent than the never-treated community sample and almost all the difference between the groups could be accounted for by active symptoms. A further study revealed that specific threat/ control override symptoms largely explained the relationship. These threat/ control override symptoms represent experiences of patients feeling that people are trying to harm them and experiences of their minds being dominated by forces outside their control. These results have been replicated subsequently (Swanson et al, 1990, 1996, 1997; Link et al, 1998). The data in these studies, however, have been criticised for being retrospective, having been gathered for other purposes and having weak measures of delusions and violence. The MacArthur Violence Risk Assessment Study has largely overcome these methodological limitations and casts doubt on the importance of threat/ control override delusions as mediators for violence (Appelbaum et al, 2000). $\mathrm{N}$ either delusions in general nor threat/ control override delusions in particular were found to be associated with an increased risk of violence in this study. 
The authors suggest that the reliance on self-report in previous studies may have resulted in the mislabelling of other phenomena that can contribute to violence as delusions

\section{RISK OF VIOLENCE IN SOCIETY}

M ost research to date has examined the association between violence and schizophrenia in terms of relative risk (i.e. the amount of risk posed by those with schizophrenia relative to others). Surprisingly little work, however, has focused on the more important public health issue of population-attributable risk per cent (PAR\%): the percentage of violence in the population that can be ascribed to schizophrenia and thus could be eliminated if schizophrenia was eliminated from the population. It is possible to perform approximate calculations of the PAR \% on data reported from some population-based studies. To calculate this figure, we first calculate the rate of violence in the total study population and then subtract the rate of violence in the population by removing all those with schizophrenia. This figure then is divided by the rate in the total population and multiplied by 100 . In the ECA study in America, $2.7 \%$ of individuals who reported community violence over one year had schizophrenia (Swanson et al, 1990). In a Finnish cohort study, those with schizophrenia accounted for $4 \%$ of those registered for at least one violent crime (Tiihonen et al, 1997). In a Danish birth cohort followed to age 44 years, $2 \%$ of all males with lifetime arrests for violence and $9 \%$ of all females had schizophrenia. When we exclude those with comorbid substance misuse, these figures drop to $0.8 \%$ for males and $6 \%$ for females (Brennan et al, 2000). In Dunedin, New Zealand, $94 \%$ of a total city birth cohort were followed up at age 21 years. Without considering comorbidity, just over $10 \%$ of past-year violencecommitted by theseyoung adults was attributable to schizophrenic spectrum disorders.

M ost of the above figures represent a fairly small percentage of the total violence in these populations. The problem is that the PAR\% assumes that causality has been established. It thus fails to take into account other risk factors or confounding factors that may be operating in the association between a particular risk factor and disease. As we have seen previously, for example, comorbidity substantially increases the risk of violence in schizophrenia and it is thus possible that if substance abuse was to be eliminated from the population, the contribution to violence made by schizophrenia alone would be much less.

To prevent unnecessary stigmatization of the seriously mentally ill, it is the duty of researchers to present a balanced picture. By neglecting to report measures of both relative and absolute risk, a skewed picture may emerge. One example of a balanced report found that men with schizophrenia were up to five times more likely to be convicted of serious violence than the general population (Wallace et al, 1998). Results also presented indicated that $99.97 \%$ of those with schizophrenia would not be convicted of serious violence in a given year and that the probability that any given patient with schizophrenia will commit homicide is tiny (approximate annual risk is 1:3000 for men and 1:33 000 for women).

Risk is generally presented in terms of odds ratios, yet research has shown that people find it difficult to digest such measures. Better ways are required for presenting risk magnitudes in a digestible form, and a logarithmic scale provides the basis for a common language for describing risk (Calman \& Royston, 1997). It has been suggested that community risk scales that describe the magnitude of risk in relation to an individual's community may be most useful. If communities are grouped into roughly logarithmic clusters (e.g. individual (1), family (10), village (1000), etc.), then such a classification allows individuals to think in terms of level of risk to themselves, their family, their town and so forth. This system also allows a consideration of how the risk of violence by people with schizophrenia compares with other risks, if also presented in the same way.

\section{CONCLUSION}

The weight of the evidence to date is that although a statistical relationship does exist between schizophrenia and violence, only a small proportion of societal violence can be attributed to persons with schizophrenia. Future research should focus on the interplay of various factors affecting this relationship by using robust methodologies (i.e. multiple measurements of violence and avoidance of bias).

\section{REFERENCES}

Appelbaum, P. S., Robbins, P. C. \& Monahan, J. (2000) Violence and delusions: data from the MacArthur Violence Risk Assessment Study. American Journal of Psychiatry, 157, 566-572.

Arboleda-Florez, J., Holley, H. \& Crisanti, A. (1998) Understanding causal paths between mental illness and violence. Social Psychiatry and Psychiatric Epidemiology, 33, S38-S46.

Arseneault, L., Moffitt, T. E., Caspi, A., et al (2000) Mental disorders and violence in a total birth cohort: results from the Dunedin study. Archives of General Psychiatry, 57, 979-968.

Brennan, P. A., Mednick, S. A. \& Hodgins, S. (2000) Major mental disorders and criminal violence in a Danish birth cohort. Archives of General Psychiatry, 57, 494-500.

Calman, K. C. \& Royston, G. H. (1997) Risk language and dialects. BMJ, 315, 939-942.

Cuffel, B. J., Shumway, M., Choujian, T. L., et al (1994) A longitudinal study of substance use and community violence in schizophrenia. Journal of Nervous and Mental Disease, 182, 704-708.

Eaton, w. \& Kessler, L. (1985) The NIMH

Epidemiologic Catchment Area Study. Epidemiological Field Methods in Psychiatry. New York: Academic Press.

Elliott, D., Huizinga, D. \& Morse, B. (1986) Selfreported violent offending: a descriptive analysis of juvenile violent offenders and their offending careers. Journal of Interpersonal Violence, I, 472-513.

Eronen, M., Angermeyer, M. C. \& Schulze, B. (1998) The psychiatric epidemiology of violent behaviour. Social Psychiatry and Psychiatric Epidemiology, 33, SI3-S23.

_ , Hakola, P. \& Tiihonen, J. (1996) Mental disorders and homicidal behaviour in Finland. Archives of General Psychiatry, 53, 497-50I.

Harris, G., Rice, M. \& Quinsey, V. (1993) Violent recidivism of mentally disordered offenders: the development of a statistical prediction instrument. Criminal Justice and Behavior, 20, 315-335.

Hodgins, S. (1992) Mental disorder, intellectual deficiency, and crime: evidence from a birth cohort. Archives of General Psychiatry, 49, 476-483.

_, Mednick, S. A., Brennan, P. A., et al (1996) Mental disorder and crime: evidence from a Danish birth cohort. Archives of General Psychiatry, 53, 489-496.

Humphreys, M. S., Johnstone, E. C., MacMillan, J. F., et al (1992) Dangerous behaviour preceding first admissions for schizophrenia. British Journal of Psychiatry, 16I, 50I-505.

Karson, C. \& Bigelow, L. B. (1987) Violent behaviour in schizophrenic patients. Journal of Nervous and Mental Disease, 175, 161-164.

Lindqvist, P. \& Allebeck, P. (1990) Schizophrenia and crime. A longitudinal follow-up of 644 schizophrenics in Stockholm. British journal of Psychiatry, 157, 345-350.

Link, B. G., Andrews, H. \& Cullen, F. T. (1992) The violent and illegal behaviour of mental patients reconsidered. American Sociological Review, 57, 275-292.

_, Stueve, A. \& Phelan, J. (1998) Psychotic symptoms and violent behaviors: probing the components of 'threat/control-override' symptoms. Social Psychiatry and Psychiatric Epidemiology, 33, S55-S60. 
Monahan, J. (1997) Clinical and Actuarial predictions of violence. In Modern Scientific Evidence: The Law and Science of Expert Testimony (eds D. Faigman, D. Kaye M. Saks, et al) Vol. I, pp. 300-318. St Paul, MN: West Publishing Company.

_ \& Appelbaum, P. (2000) Reducing violence risk: diagnostically based clues from the MacArthur Violence Risk Assessment Study. In Effective Prevention of Crime and Violence among the Mentally III (ed. S. Hodgins), pp. 19-34. The Netherlands: Kluwer Academic Publishers.

Mullen, P. E. (1997) A reassessment of the link between mental disorder and violent behaviour, and its implications for clinical practice. Australian and New Zealand Journal of Psychiatry, 3I, 3-1I.

_ , Burgess, P., Wallace, C., et al (2000) Community care and criminal offending in schizophrenia. Lancet, $\mathbf{3 5 5}$, 614-617.

Mulvey, E. P., Shaw, E. \& Lidz, C. W. (1994a) Why use multiple sources in research on patient violence in the community. Criminal Behaviour and Mental Health, 4, 235-258.

_ _ _ \& _ (1994b) Assessing the evidence of a link between mental illness and violence. Hospital and Community Psychiatry, 45, 663-668.

Robins, L. N. \& Reiger, D. A. (1991) Psychiatric Disorders in America. New York, NY: The Free Press.

Steadman, H. J., Mulvey, E. P., Monahan, J., et al (1998) Violence by people discharged from acute psychiatric inpatient facilities and by others in the same neighborhoods. Archives of General Psychiatry, 55, I-9.

Stueve, A. \& Link, B. G. (1997) Violence and psychiatric disorders: results from an epidemiological study of young adults in Israel. Psychiatry Quarterly, 68, 327-342.

Swanson, J. W., Borum, R. \& Swartz, M. S. (1996) Psychotic symptoms and disorders and the risk of violent behaviour in the community. Criminal Behaviour and Mental Health, 6, 309-329.

_, Estroff, S., Swartz, M., et al (1997) Violence and severe mental disorder in clinical and community populations: the effects of psychotic symptoms, comorbidity, and lack of treatment. Psychiatry, 60, 1-22.

_, Holzer, C. E. III, Ganju, V. K., et al (1990) Violence and psychiatric disorder in the community: evidence from the Epidemiologic Catchment Area surveys. Hospital and Community Psychiatry, 4I, 76I-770.

Taylor, P. J. (1985) Motives for offending among violent and psychotic men. British Journal of Psychiatry, 147 491-498.

- (1998) When symptoms of psychosis drive serious violence. Social Psychiatry and Psychiatric Epidemiology, 33, S47-S54.

_\& Gunn, J. (1984) Violence and psychosis I. Risk of violence among psychotic men. BM], 288, 1945-1949.

\section{CLINICAL IMPLICATIONS}

- There is a significant association between violence and schizophrenia, but less than $10 \%$ of societal violence is attributable to schizophrenia. Comorbid substance abuse considerably increases the risk.

- The lack of methodological consistency on which the association is based underlines the need for standard definitions of exposure and outcome and comparable designs across studies.

- In reporting the association between violence and schizophrenia, a shift of focus from the relative risk to the absolute risk posed to the community should reduce stigma.

\section{LIMITATIONS}

- In the interests of space and clarity, citation of the literature is selective.

- Estimates of population-attributable risk are approximate and are limited to the few population studies that provide sufficient data for their calculation.

- Little attention is given to the interplay of risk factors for violence in schizophrenia.

ELIZABETH WALSH, MRCPsych, Section of Forensic Mental Health, Institute of Psychiatry, London; ALEC BUCHANAN, MD, Department of Forensic Psychiatry, Institute of Psychiatry, London; THOMAS FAHY, MD, Section of Forensic Mental Health, Institute of Psychiatry, London

Correspondence to: Dr Elizabeth Walsh, Section of Forensic Mental Health, Institute of Psychiatry, De Crespigny Park, Denmark Hill, London SE5 8AF. E-mail: sppmemw@op.kcl.ac.uk

(First received 8 March 200I, final revision 14 August 200I, accepted I4 August 200I)

_ \& _ (1999) Homicides by people with mental illness: myth and reality. British Journal of Psychiatry, 174, 9-14

Teplin, L. A. (1990) The prevalence of severe mental disorder among urban jail detainees: comparison with the epidemiologic catchment area program. American Journal of Public Health, 80, 663-669.

\section{Tiihonen, J., Isohanni, M., Rasanen, P., et al (1997)} Specific major mental disorders and criminality: a 26-year prospective study of the 1966 northern Finland birth cohort. American Journal of Psychiatry, I54 $840-845$

Volavka, J., Laska, E., Baker, S., et al (1997) History of violent behaviour and schizophrenia in different cultures.
Analyses based on the WHO study on Determinants of Outcome of Severe Mental Disorders. British Journal of Psychiatry, 17I, 9-14.

Walker, Z. \& Seifert, R. (1994) Violent incidents in a psychiatric intensive care unit. British Journal of Psychiatry, 164, 826-828.

Wallace, C., Mullen, P., Burgess, P., et al (1998)

Serious criminal offending and mental disorder. Case linkage study. British Journal of Psychiatry, 172, 477-484.

Wessely, S. C., Castle, D., Douglas, A. J., et al (1994) The criminal careers of incident cases of schizophrenia. Psychological Medicine, 24, 483-502. 